
\title{
28 Research Suare \\ Efficacy and Safety of Remdesivir in COVID-19 Patients: A Retrospective Analysis of Active Surveillance Database
}

Vaishali Gupte ( $\boldsymbol{\alpha}$ dr.gupte@Cipla.com )

Cipla Ltd. https://orcid.org/0000-0003-4439-2416

\section{Rashmi Hegde}

Cipla Ltd.

\section{Sandesh Sawant}

Cipla Ltd.

Kabil Kalathingal

Cipla Ltd.

Sonali Jadhav

Cipla Ltd.

\section{Rohit Malabade}

Cipla Ltd.

Jaideep Gogtay

Cipla Ltd.

\section{Research article}

Keywords: Remdesivir, COVID-19, Retrospective studies, Retrospective analysis, Active surveillance

Posted Date: March 31st, 2021

DOl: https://doi.org/10.21203/rs.3.rs-366384/v1

License: (9) (i) This work is licensed under a Creative Commons Attribution 4.0 International License.

Read Full License 


\section{Abstract}

Background: Real-world data on safety and efficacy of remdesivir in COVID-19 management is scant. We present findings of data analysis conducted for assessing the clinical outcomes of remdesivir treatment for COVID-19 in India.

Methods: This retrospective analysis used data from an active surveillance programme database of hospitalised patients with COVID-19 who were receiving remdesivir.

Results: Of the 2329 patients included, $67.4 \%$ were men. Diabetes $(29.69 \%)$ and hypertension $(20.33 \%)$ were the most common comorbidities. At remdesivir initiation, $2272(97.55 \%)$ patients were receiving oxygen therapy. Remdesivir was administered for 5 days in $65.38 \%$ of patients. Antibiotics $(64.9 \%)$ and steroids (47.9\%) were the most common concomitant medications. Remdesivir was overall well tolerated: $13 \%$ of patients reported 119 adverse events; most common were nausea and vomiting in $45.4 \%$ and increased liver enzymes in $14.28 \%$ patients. $84 \%$ of patients were cured/improved, $6.02 \%$ died, and $9.16 \%$ showed no improvement in their clinical status at data collection. Subgroup analysis showed that the mortality rate was significantly lower in patients $<60$ years old than in those $>60$ years old. Amongst patients on oxygen therapy, the cure/improvement rate was significantly higher in those receiving standard low-flow oxygen than in those receiving mechanical ventilation, non-invasive ventilation, or highflow oxygen. Risk factors for higher mortality were age > 60 years, hypertension, cardiac disease, diabetes, and mechanical ventilation.

Conclusion: Our analysis showed that remdesivir is well tolerated and has an acceptable safety profile. The cure/improvement rate was $84 \%$, with a higher improvement in patients $<60$ years old and on standard low-flow oxygen.

\section{Introduction}

Coronavirus disease 2019 (COVID-19) is a novel respiratory disease caused by severe acute respiratory syndrome coronavirus-2 (SARS-CoV-2). In March 2020, the COVID-19 outbreak was declared as a global pandemic by the World Health Organization (WHO) [1]. As of 28 December 2020, the global incidence of COVID-19 had reached 79,232,555 confirmed cases; India was the second most-affected country in the world, with a case burden of 10,187,850 confirmed cases. As the pathogenesis underlying COVID-19 became more apparent, global strategies evaluating therapeutic options, including new antivirals, evolved rapidly. However, repositioning the already existing therapeutics remained a commonly adapted strategy recommended by the WHO [2].

Remdesivir is an adenosine analogue with broad-spectrum antiviral activity against several singlestranded RNA viruses. It was originally developed for treating patients with Ebola virus infection [3]. After recording the potential benefits of remdesivir against SARSCoV-2 in in vitro, pre-clinical, and human cell line studies, its efficacy was evaluated in patients with COVID-19 [4-6]. On 1 May 2020, remdesivir received the Emergency Use Authorisation (EUA) status based on a preliminary report from an interim 
analysis of an ongoing double-blind randomised controlled trial by the United States Food Drug Administration (US FDA) [7]. On 21 June 2020, the Central Drugs Standard Control Organisation (CDSCO) approved its restricted emergency use for treating patients with severe COVID-19 infection in India; the indication was later expanded to moderate and severe disease. However, the CDSCO approved remdesivir with a condition to provide data from an active surveillance programme on a monthly basis by the pharmaceutical manufacturers [7]. Given the global emergency and the unmet medical need with respect to COVID-19 treatment, the Drugs Controller General of India (DCGI) also provided a clinical trial waiver for remdesivir use in India [7]. At the time of writing this paper, clinical evidence for its safety and efficacy in COVID-19 pertains mainly to randomised trials, and only few observational data are available that show its safety in real practice. In this paper, we present a retrospective analysis of data from an active surveillance programme conducted for remdesivir use in patients with COVID-19 in India.

\section{Methods}

\section{Surveillance design and participants}

This retrospective analysis evaluated active surveillance data of hospitalised patients with COVID-19 who received remdesivir treatment (Cipremi ${ }^{\circledR}$; Cipla Ltd). Remdesivir was administered to patients with COVID19 at participating hospitals in accordance with the restricted emergency use approval for remdesivir by CDSCO, India.

We retrospectively analysed the data of patients who had received remdesivir therapy from July 2020 until October 2020, with an aim to evaluate its safety and efficacy. All hospitals that administered remdesivir in COVID-19 patients were obliged to provide data through an active surveillance form.

\section{Procedure and outcomes}

The physician/clinical staff filled an online surveillance form (Fig. S1) for each patient with suspected or confirmed COVID-19 who was administered remdesivir. Data regarding patient age, gender, comorbid conditions, concomitant medicines, status of oxygen supplementation, remdesivir treatment duration, and any adverse event were collected. Clinical outcomes were defined as cure (complete resolution of symptoms), improvement, no improvement, or death.

\section{Statistical analysis}

Continuous and quantitative variables are summarised using descriptive statistics. Categorical data are presented as frequency count $(N)$ and percentages (\%). All statistical analyses were performed using the Statistical Package for the Social Sciences version 23.0. A subgroup analysis was performed to assess the association of clinical and demographic characteristics with the clinical outcomes. Patients with missing information for a given variable were excluded from the calculations/analysis. A $p$ value of $<$ 0.05 was considered statistically significant. 


\section{Patient demographics}

Data of 2329 patients were available through the online or paper-based active surveillance log from July 2020 up to 15 October 2020. The geographical distribution showed that Tamil Nadu (29.4\%), Telangana (11.1\%), Uttar Pradesh (10\%), West Bengal (9.3\%), and Maharashtra (8.8\%) contributed maximally to the current data. Most patients were in the age group of $40-60$ years (49.9\%) followed by the age group of > 60 years (33.7\%). Men comprised $67.4 \%$ of the analysed patient population (Table 1 ).

Table 1

Patient demographics

\begin{tabular}{|ll|}
\hline Characteristics & $\mathbf{N ( \% )}$ \\
\hline Age group (years) & \\
\hline$<12$ & $2(0.1)$ \\
\hline$\geq 12$ to $<20$ & $5(0.2)$ \\
\hline$\geq 20$ to $<40$ & $376(16.1)$ \\
\hline$\geq 40$ to $<60$ & $1162(49.9)$ \\
\hline$\geq 60$ & $784(33.7)$ \\
\hline Gender & \\
\hline Male & $1570(67.4)$ \\
\hline Female & $583(25)$ \\
\hline Gender not disclosed & $176(7.6)$ \\
\hline
\end{tabular}

\section{Clinical characteristics}

Up to $98.2 \%$ of patients had comorbid conditions. Diabetes $(29.69 \%)$ was the most common comorbid condition followed by hypertension (20.33\%), cardiac diseases (6.34\%), and lung disease $(4.37 \%)$ (Fig. 1a). In addition to these comorbidities, $44.82 \%$ had other diseases such as blood cancer, cholangitis, and chronic kidney disease. A total of 2,272 (97.55\%) patients were receiving oxygen therapy at the time of starting remdesivir: the most common oxygen supplementation method was standard lowflow oxygen (65.27\%), followed by high-flow oxygen (19.06), non-invasive ventilation (12.02), and mechanical ventilation (3.52\%) (Fig. 1b). Duration of remdesivir administered in $65.38 \%$ of patients was 5 days, while $12 \%$ of patients received remdesivir for $\geq 6$ days. Among 1081 patients on concomitant medications, antibiotics were the most common concomitant medications ( $64.9 \%$ of the patients) followed by steroids (47.9\% of the patients) (Fig. 1c).

\section{Adverse events and safety}


A total of 119 adverse events were reported in $13 \%$ of patients; most common were nausea and vomiting (45.4\%) followed by increased liver enzyme levels (increased serum glutamic pyruvic transaminase [SGPT], serum glutamic oxaloacetic transaminase [SGOT] levels) (14.28\%), rash (5.8\%), bradycardia (2.5\%), nephrotoxicity (1.68\%), and oral ulcer ( $0.8 \%)$.

\section{Clinical outcomes}

The clinical outcome of cure or improvement was recorded in $84 \%$, death in $6.02 \%$, and no improvement in $9.16 \%$ of the patients at the time of data collection (Fig. 2).

\section{Subgroup analysis}

Results of various subgroup analyses are presented in Table 2. When clinical outcomes were analysed by patient age, cure/improvement rate was significantly higher in the age groups of $20-40$ years $(91.45 \%, p$ $<0.0001)$ and $40-60$ years $(85.33 \%, p=0.0011)$ compared with that in the age group of $\geq 60$ years (78.99\%). Similarly, the cure/improvement rate was significantly higher in the age group of 20-40 years compared with that in the age group of $40-60$ years $(91.45 \%$ vs $85.33 \%, p=0.0083)$. Mortality rate was higher in patients $\geq 60$ years old compared with those $20-40$ years old $(10.07 \%$ vs $3.62 \%, p<0.0001)$ and $40-60$ years old $(10.07 \%$ vs $5.37 \%, p=0.0004)$. 
Table 2

Subgroup analysis of clinical outcomes by clinical and demographic characteristics

Characteristics

$N(\%)$

Cure/improvement

Death/

No improvement

death related to COVID-19

Age group (years)

$\begin{array}{llll}12-20 & 4(80.00) & 0(0.00) & 1(20.00) \\ 20-40 & 278(91.45)^{\# \star} & 11(3.62)^{\# \# \star \star} & 15(4.93)^{\# \# \# \star \star \star} \\ 40-60 & 826(85.33)^{\$} & 52(5.37)^{\$ \$} & 90(9.30)^{\$ \$ \$} \\ \geq 60 & 549(78.99) & 70(10.07) & 75(10.79)\end{array}$

Gender

Male

1,206(83.29)@

$108(7.46) @$

$134(9.25) @$

Female

451 (86.07)

$26(4.96)$

47 (8.97)

Diabetes

Yes

489 (78.36)

62 (9.93)

$72(11.54)$

No

$1,136(85.52)$

$71(5.41)$

$106(8.07)$

Hypertension

Yes

377 (87.06)

23 (5.32)

$33(7.62)$

No

$1,248(82.98)$

$110(7.31)$

145 (9.64)

Other cardiac conditions

Yes

105 (81.40)

19 (14.73)

$5(3.88)$

No

$1,520(84.07)$

$114(6.31)$

173 (9.57)

Lung disease

Note: The patient status "No improvement" in the last column was reported at the time of data entry which can eventually change.

SLFO, standard low-flow oxygen; HFO, high-flow oxygen; NIV, non-invasive ventilation; MV, mechanical ventilation

${ }^{\#} p=0.0083$ vs $40-60$ years; * $p<0.0001$ vs $>60$ years; ${ }^{\$} p=0.0011$ vs $>60$ years; ${ }^{\# \#} p=0.2812$ vs $40-60$ years; $* \star p<0.0001$ vs $>60$ years; $\$ \$ p=0.0004$ vs $>60$ years; \#\#\# $p=0.0219$ vs $40-60$ years;

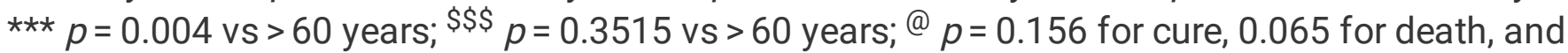
0.916 for no improvement; @ @ $p=0.112$ compared with patients not receiving steroids 


\begin{tabular}{|c|c|c|c|}
\hline \multirow[t]{2}{*}{ Characteristics } & \multicolumn{3}{|l|}{$N(\%)$} \\
\hline & \multicolumn{3}{|c|}{ death related to COVID-19 } \\
\hline Yes & $72(77.42)$ & $10(10.75)$ & $11(11.83)$ \\
\hline No & $1,553(84.22)$ & $123(6.67)$ & $167(9.06)$ \\
\hline \multicolumn{4}{|l|}{ Received oxygen support } \\
\hline Yes & $1,608(83.62)$ & $134(6.97)$ & $181(9.41)$ \\
\hline No & $37(100.00)$ & $0(0.00)$ & $0(0.00)$ \\
\hline SLFO & 1,107 (93.97) & $26(2.21)$ & $45(3.82)$ \\
\hline HFO & $332(80.58)$ & $24(5.82)$ & $56(13.59)$ \\
\hline NIV & $146(57.71)$ & $48(18.97)$ & $59(23.32)$ \\
\hline MV & $23(28.75)$ & $36(45.00)$ & $21(26.25)$ \\
\hline Received steroids & 383 (79.95) @@ & $40(8.35) @ @$ & 56 (11.69)@@ \\
\hline Received antibiotics & $456(79.31)$ & $52(9.05)$ & $67(11.65)$ \\
\hline Received anticoagulants & $253(81.35)$ & $19(6.11)$ & $39(12.54)$ \\
\hline \multicolumn{4}{|c|}{$\begin{array}{l}\text { Note: The patient status "No improvement" in the last column was reported at the time of data entry } \\
\text { which can eventually change. }\end{array}$} \\
\hline \multicolumn{4}{|c|}{$\begin{array}{l}\text { SLFO, standard low-flow oxygen; HFO, high-flow oxygen; NIV, non-invasive ventilation; MV, mechanical } \\
\text { ventilation }\end{array}$} \\
\hline \multicolumn{4}{|c|}{ 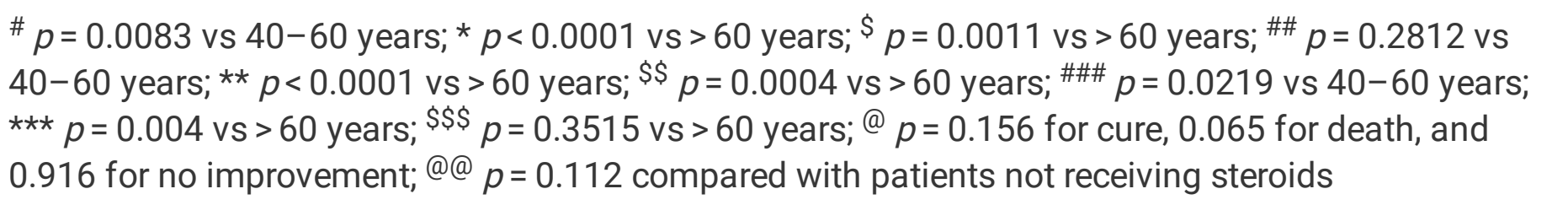 } \\
\hline
\end{tabular}

Although the mortality rate was slightly higher among the older adults (>60 years), difference in the mortality rate remained statistically non-significant for the age groups of $40-60$ years and $20-40$ years $(5.37 \%$ vs $3.62 \% ; p<0.2812)$. The clinical outcomes were not different between men and women $(p>$ 0.05). Furthermore, similar cure/improvement rates were observed irrespective of comorbid conditions (diabetes: $50 \%$, hypertension: $59 \%$, cardiac diseases: $58 \%$, lung disease: $57 \%$ ). Mortality rate was significantly lower among those who received standard low-flow oxygen $(2 \%)$ compared with those who received mechanical ventilation $(45 \%, p<0.0001)$, noninvasive ventilation $(19 \%, p<0.0001)$, or high-flow oxygen $(6 \%, p<0.001)$. Interestingly, mortality rate was not different between patients who received and those who did not receive concomitant steroids $(p=0.112)$. 
Another subgroup analysis assessed the factors associated with the incidence of death (Table 3). The odds of death were 2.98 times higher in patients $\geq 60$ years old compared with patients 20-40 years old. Comorbidities such as cardiac disease and diabetes increase the odds of death by 1.93 and 2.5 times compared with absence of these comorbidities, respectively. Similarly, the odds of death were higher amongst patients receiving high-flow oxygen, non-invasive ventilation, or mechanical ventilation compared with standard low-flow oxygen.

Table 3

Factors associated with mortality rate

\begin{tabular}{|c|c|c|c|}
\hline Variables & Odds ratio & 95\% Confidence interval & $p$-value \\
\hline \multicolumn{4}{|l|}{ Age group (years) } \\
\hline $20-40$ & 1 & & \\
\hline $40-60$ & 1.512 & $(0.779,2.936)$ & 0.222 \\
\hline$\geq 60$ & 2.988 & $(1.559,5.728)$ & 0.001 \\
\hline \multicolumn{4}{|l|}{ Gender } \\
\hline Male & 1.544 & $(0.99,2.398)$ & 0.062 \\
\hline Female & 1 & & \\
\hline \multicolumn{4}{|l|}{ Co-morbidities } \\
\hline Hypertension (Yes/No) & 0.71 & $(0.447,1.128)$ & 0.146 \\
\hline Diabetes (Yes/No) & 1.93 & $(1.36,2.75)$ & 0.000 \\
\hline Lung disease (Yes/No) & 1.845 & $(0.959,3.559)$ & 0.063 \\
\hline Cardiac (Yes/No) & 2.565 & $(1.52,4.33)$ & 0.000 \\
\hline \multicolumn{4}{|l|}{ Type of Oxygen Support } \\
\hline SLFO & 1 & & \\
\hline $\mathrm{HFO}$ & 2.74 & $(1.55,4.83)$ & 0.000 \\
\hline NIV & 10.37 & $(6.29,17.10)$ & 0.000 \\
\hline MV & 36.25 & $(20.14,65.23)$ & 0.000 \\
\hline
\end{tabular}

\section{Discussion}

In this retrospective analysis of data from an active surveillance programme, we assessed efficacy and safety of remdesivir by measuring the clinical outcomes (cure, improvement, no improvement, or death) 
in hospitalised patients with COVID-19 who were treated with remdesivir, and their subgroups. The cure/improvement rate was $84 \%$, which is in accordance with the cure/improvement reported in historical cohorts [8-15]. Most patients were male, were in the age group of 40-60 years, required oxygen therapy, and received remdesivir for 5 days. Diabetes and hypertension were the most common comorbidities. Low cure rate and high mortality was seen in patients $>60$ years old.

Remdesivir is a nucleotide prodrug effective against various RNA viruses such as Nipah virus, respiratory syncytial virus, Ebola virus, SARS-CoV-1, and the Middle East respiratory syndrome coronavirus (MERSCoV) [16]. Remdesivir is metabolised intracellularly to its ATP analogue, which inhibits viral RNA polymerase and thus halts viral replication [3]. Remdesivir emerged as a candidate drug in the current COVID-19 pandemic and received full approval for use in COVID-19 by the USFDA $[3,17]$. Remdesivir was well tolerated in clinical trials and in the compassionate-use programme. Common adverse events reported were nausea, elevated ALT levels, headache, hypokalaemia, worsening respiratory failure, and constipation $[8-10,12,13]$. In our analysis, $13 \%$ patients reported adverse events, with nausea and increased liver enzyme levels being the most common.

In the randomised, controlled ACTT-1 trials, patients in the remdesivir group were significantly more likely to have clinical improvements than those in the placebo group $[8,10]$. In a robust pooled analysis that included patients enrolled in the SIMPLE-severe trial and a retrospective cohort of severe COVID-19 patients receiving standard-of-care, recovery rate was $74.4 \%$ on day 14 [11]. An improvement in the clinical status by at least 2 points on the ordinal scale (or being discharged alive) was seen in $71.9 \%$ of the patients and that by $\geq 1$ points was seen in $76.2 \%$ of the patients on day 14 [11]. The cure/improvement rate in our analysis was $84 \%$.

In the SIMPLE-severe trial, patients randomised to a 5- or 10-day course of remdesivir did not show a significant difference. Our analysis showed that most patients received a 5-day course of remdesivir therapy, which is in line with the DCGI-approved prescribing information.

The mortality rate following 5 days of treatment with remdesivir was $8 \%$ on day 14 in the SIMPLE-severe trial and $1 \%$ on day 28 in the SIMPLE-moderate study $[8,9]$. Following 10 days of treatment with remdesivir, the mortality rate by day 15 was $6.7 \%$ in the ACTT- 1 trial and $2 \%$ by day 14 in the SIMPLEsevere trial $[9,10]$. The WHO Solidarity trial, which included 11,330 patients from 30 countries, showed no improvement in the mortality rate in patients randomised to remdesivir treatment compared with the local standard-of-control. In another pooled analysis, the mortality rate was $7.6 \%$ on day 14 [18]. In our analysis, the mortality rate was $6.02 \%$.

During the COVID-19 pandemic, several studies reported that older adults and those with comorbid hypertension, diabetes, obesity, and heart disease are at higher risk for developing life-threatening COVID19 illness $[13,19]$. In the current analysis, remdesivir therapy showed similar cure/improvement rate irrespective of comorbid conditions. However, numerically higher mortality was observed in patients with cardiac disease $(12 \%)$, followed by that in lung disease (11\%), diabetes (8\%), and hypertension (4\%). Lower grade of respiratory support and age $<65$ years were associated with a $>2$ point improvement on 
the ordinal scale in patients treated with remdesivir [20]. Our results showed higher patient cure/improvement rate when patients did not require oxygen support and were $<60$ years of age. An Indian retrospective study, the SORT trial enrolled 350 patients treated with remdesivir and showed that patients who received remdesivir early (within 9 days of symptom onset) were more likely to have a lower incidence of mortality compared with those treated after $\geq 9$ days of symptom onset, suggesting that initiating remdesivir earlier during the disease course in moderate-to-severe COVID-19 infection may show better clinical improvements/outcomes [15].

Our analysis had some limitations as well. This was a retrospective analysis of the data obtained from an active surveillance programme database; therefore, comparison of the results with the control group could not be performed. Moreover, this analysis could not detect the association using multivariate analysis. Cure/improvement rates were not defined in terms of the ordinal scale. Data was collected at a single time point. However, our analysis presents findings of a large cohort of COVID-19 patients treated with remdesivir in real-life clinical settings in India and adds to the clinical evidence on remdesivir use in COVID-19.

\section{Conclusion}

The retrospective analysis of data from an active surveillance programme of remdesivir therapy in patients with COVID-19 showed that remdesivir was well tolerated and had an acceptable safety profile. The cure/improvement rate was $84 \%$, with greater cure rate in patients with age $<60$ years and receiving standard low-flow oxygen.

\section{Abbreviations}

COVID-19: Coronavirus disease 2019; SARS-CoV-2: severe acute respiratory syndrome coronavirus-2; WHO: World Health Organization; US FDA: United States Food Drug Administration; CDSCO: Central Drugs Standard Control Organisation; DCGI: Drugs Controller General of India; SGPT: serum glutamic pyruvic transaminase; SGOT: serum glutamic oxaloacetic transaminase; MERS-CoV: Middle East respiratory syndrome coronavirus.

\section{Declarations}

\section{Ethics approval and Consent to participate}

All hospitals and clinics that administered remdesivir in COVID-19 patients were obliged to provide data through an active surveillance form thus, obtaining ethical approval and informed consent is not required.

\section{Consent for publication}

Not applicable. 


\section{Competing interests}

All the authors are permanent employees of Cipla, Ltd.

\section{Funding}

The analysis was funded by Cipla Ltd., India.

\section{Authors' contributions}

All authors contributed equally to the study conception, design, data collection and analysis. All the authors had equal contribution in drafting the manuscript or revising it critically for important intellectual content. All authors read and approved the final manuscript.

\section{Acknowledgements}

The authors thank $\mathrm{CBCC}$ Global Research for providing medical writing assistance for this manuscript. Authors would like to acknowledge Chest Research and Training Pvt Ltd. (CREST) for providing statistical analysis support for this manuscript. The authors also thank the staff of hospitals and healthcare professionals who have participated in the data collection and analysis.

\section{Availability of data and material}

There are no associated data other than that included in the manuscript.

\section{Author details}

${ }^{1}$ Director, Medical Services, Cipla Ltd., Mumbai, India. ${ }^{2}$ Vice President, Medical Services, Cipla Ltd., Mumbai, India. ${ }^{3}$ Senior Director, Head Clinical Research, Cipla Ltd., Mumbai, India. ${ }^{4}$ Associate Director, Drug Safety, Cipla Ltd., Mumbai, India. ${ }^{5}$ Manager, Medical Services - Clinical Trial Group, Cipla Ltd., Mumbai, India. ${ }^{6}$ Deputy Manager, Medical Services, Cipla Ltd., Mumbai, India. ${ }^{7}$ Executive Vice President, Global Chief Medical Officer, Cipla Ltd., Mumbai, India.

\section{References}

1. Cucinotta D, Vanelli M. WHO declares COVID-19 a pandemic. Acta Biomed. 2020;91(1):157-60. https://doi.org/10.23750/abm.v91i1.9397. 
2. Kupferschmidt $\mathrm{K}$, Cohen J. WHO launches global megatrial of the four most promising coronavirus treatments. Science. 2020. https://www.sciencemag.org/news/2020/03/who-launches-globalmegatrial-four-most-promising-coronavirus-treatments. Accessed 17 March 2021.

3. Pardo J, Shukla AM, Chamarthi G, Gupte A. The journey of remdesivir: from Ebola to COVID-19. Drugs Context. 2020;9:2020-4-14. https://doi.org/10.7573/dic.2020-4-14.

4. Wang $M$, Cao R, Zhang L, Yang $X$, Liu J, Xu M, et al. Remdesivir and chloroquine effectively inhibit the recently emerged novel coronavirus (2019-nCoV) in vitro. Cell Res. 2020;30(3):269-71. https://doi.org/10.1038/s41422-020-0282-0.

5. Williamson BN, Feldmann F, Schwarz B, Meade-White K, Porter DP, Schulz J, et al. Clinical benefit of remdesivir in rhesus macaques infected with SARS-CoV-2. bioRxiv [Preprint]. 2020;2020.04.15.043166. https://doi.org/10.1101/2020.04.15.043166.

6. Pizzorno A, Padey B, Julien T, Trouillet-Assant S, Traversier A, Errazuriz-Cerda E, et al. Characterization and treatment of SARS-CoV-2 in nasal and bronchial human airway epithelia. bioRxiv. 2020. https://doi.org/10.1101/2020.03.31.017889

7. Singh D, Wasan H, Mathur A, Gupta YK. Indian perspective of remdesivir: A promising COVID-19 drug. Indian J Pharmacol. 2020;52(7):227-8. https://doi.org/10.4103/ijp.IJP_486_20.

8. Spinner CD, Gottlieb RL, Criner GJ, Arribas López JR, Cattelan AM, Soriano Viladomiu A, et al. Effect of remdesivir vs standard care on clinical status at 11 days in patients with moderate COVID-19: a randomized clinical trial. JAMA. 2020;324(11):1048-57. https://doi.org/10.1001/jama.2020.16349.

9. Goldman JD, Lye DCB, Hui DS, Marks KM, Bruno R, Montejano R, et al. Remdesivir for 5 or 10 days in patients with severe Covid-19. N Engl J Med. 2020;383(19):1827-37. https://doi.org/10.1056/NEJMoa2015301.

10. Beigel JH, Tomashek KM, Dodd LE, Mehta AK, Zingman BS, Kalil AC, et al. Remdesivir for the treatment of Covid-19-preliminary report. N Engl J Med. 2020; 383(19):1813-26. https://doi.org/10.1056/NEJMoa2007764.

11. Olender SA, Perez KK, Go AS, Balani B, Price-Haywood EG, Shah NS, et al. Remdesivir for severe COVID-19 versus a cohort receiving standard of care. Clin Infect Dis. 2020;ciaa1041. https://doi.org/10.1093/cid/ciaa1041.

12. Grein J, Ohmagari N, Shin D, Diaz G, Asperges E, Castagna A, et al. Compassionate use of remdesivir for patients with severe Covid-19. N Engl J Med. 2020;382(24):2327-36. https://doi.org/10.1056/NEJMoa2007016.

13. Zhou F, Yu T, Du R, Fan G, Liu Y, Liu Z, et al. Clinical course and risk factors for mortality of adult inpatients with COVID-19 in Wuhan, China: a retrospective cohort study. Lancet. 2020;395(10229):1054-62. https://doi.org/10.1016/S0140-6736(20)30566-3.

14. Lapadula G, Bernasconi DP, Bellani G, Soria A, Rona R, Bombino M, et al. Remdesivir use in patients requiring mechanical ventilation due to COVID-19. Open Forum Infect Dis. 2020;7(11):ofaa481. https://doi.org/10.1093/ofid/ofaa481. 
15. Mehta RM, Bansal S, Bysani S, Kalpakam H. A shorter symptom-onset to remdesivir treatment (SORT) interval is associated with a lower mortality in moderate-to-severe COVID-19: A real-world analysis. medRxiv. 2020. https://doi.org/10.1101/2020.11.05.20226373.

16. Singh AK, Singh A, Singh R, Misra A. Remdesivir in COVID-19: A critical review of pharmacology, preclinical and clinical studies. Diabetes Metab Syndr. 2020;14(4):641-8.

https://doi.org/10.1016/j.dsx.2020.05.018.

17. US Food and Drug Administration. FDA's approval of Veklury (remdesivir) for the treatment of COVID19-The Science of Safety and Effectiveness. October 22, 2020. https://www.fda.gov/drugs/drugsafety-and-availability/fdas-approval-veklury-remdesivir-treatment-covid-19-science-safety-andeffectiveness. Accessed 17 March 2021.

18. WHO Solidarity Trial Consortium, Pan H, Peto R, Henao-Restrepo AM, Preziosi MP, Sathiyamoorthy V, et al. Repurposed antiviral drugs for COVID-19-interim WHO Solidarity trial results. N Engl J Med. 2021;384(6):497-511. https://doi.org/10.1056/NEJMoa2023184.

19. Cummings MJ, Baldwin MR, Abrams D, Jacobson SD, Meyer BJ, Balough EM, et al. Epidemiology, clinical course, and outcomes of critically ill adults with COVID-19 in New York City: a prospective cohort study. Lancet. 2020;395(10239):1763-70. https://doi.org/10.1016/S0140-6736(20)31189-2.

20. Simon Collins, HIV i-Base. Predictors of response to remdesivir in GS-5773 COVID-19 study. 2020. https://i-base.info/htb/38476. Accessed 17 March 2021.

\section{Figures}
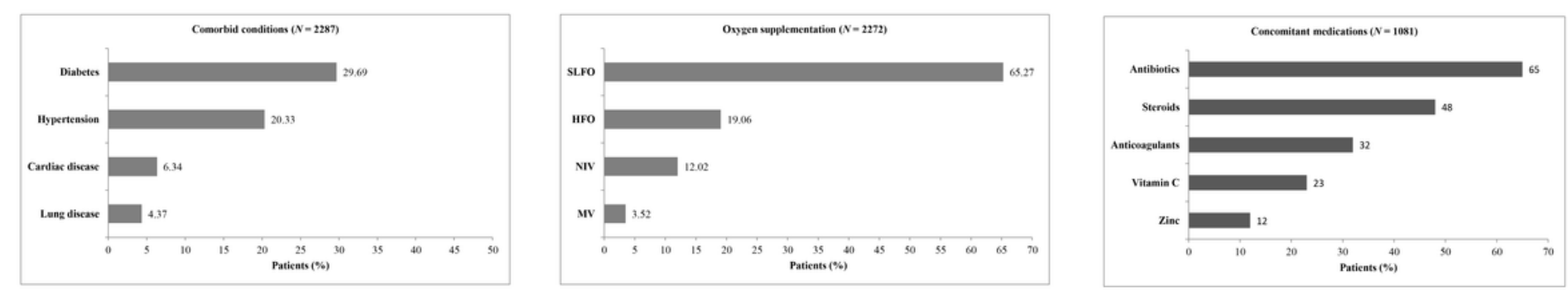

\section{Figure 1}

(a through c, respectively) represents the comorbid conditions of, status of oxygen support to, and concomitant medications administered to hospitalised patients with COVID-19 who were treated with remdesivir 1a Comorbid conditions 1b Oxygen support SLFO, standard low-flow oxygen; HFO, high-flow oxygen; NIV, non-invasive ventilation; MV, mechanical ventilation 1c Concomitant medications 


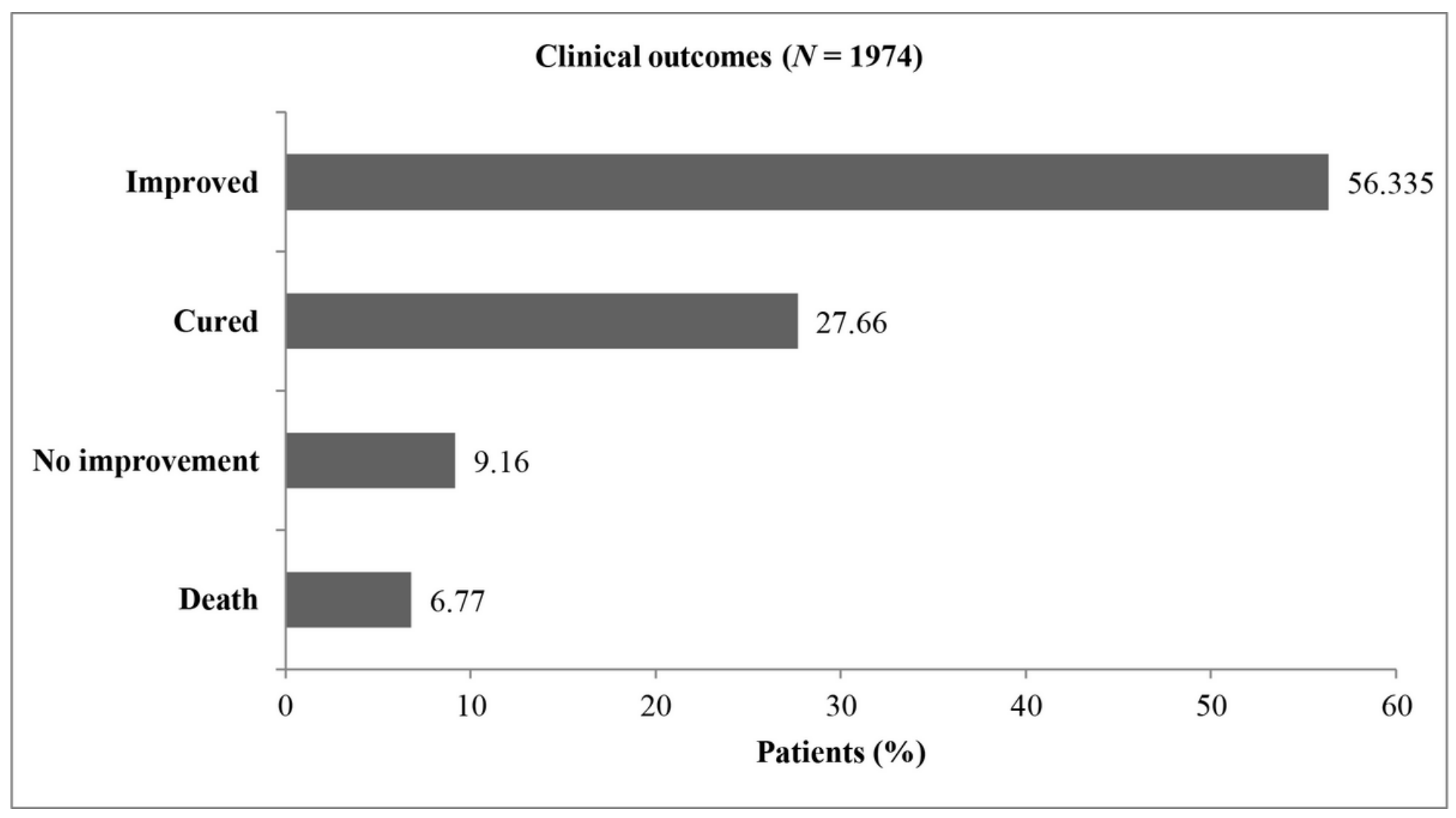

Figure 2

Clinical outcomes

\section{Supplementary Files}

This is a list of supplementary files associated with this preprint. Click to download.

- Fig.S1.tif 